\title{
CDISC SDTM Implementation Guide Version Response Terminology
}

National Cancer Institute

\section{Source}

National Cancer Institute. CDISC SDTM Implementation Guide Version Response

Terminology. NCl Thesaurus. Code C160924.

Terminology associated with the SDTM implementation guide version response codelist of the Clinical Data Interchange Standards Consortium (CDISC) Study Data Tabulation Model (SDTM). 